
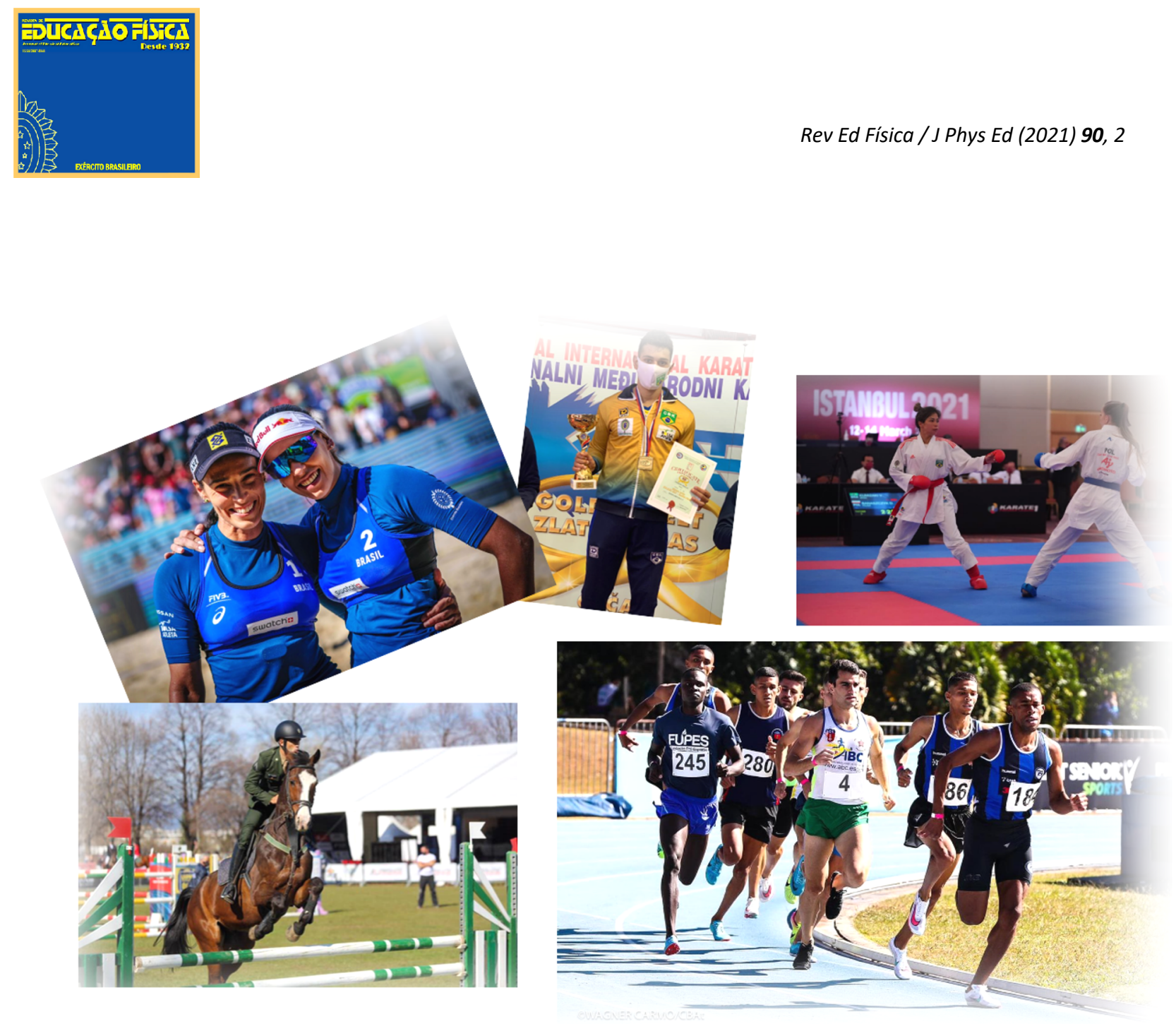

Original

\title{
A importância do Programa de Atletas de Alto Rendimento (PAAR) das Forças Armadas para o desempenho de atletas brasileiros: um estudo transversal
}

Almeida, Oliveira e Rêgo

Marcelo Lucena de Almeida ${ }^{\$ 1}$; Leonardo Perdigão de Oliveira² e

Ricardo Costa de Almeida Rêgo ${ }^{3}$ 
Artigo Original

Original Article

\section{A importância do Programa de Atletas de Alto Rendimento (PAAR) das Forças Armadas para o desempenho de atletas brasileiros: um estudo transversal}

\section{The importance of the Armed Forces High-Performance Athletes Program (PAAR) for Brazilian athletes: a Cross-Sectional Study}

Marcelo Lucena de Almeida ${ }^{\S 1}$; Leonardo Perdigão de Oliveira² MSc; Ricardo Costa de Almeida Rêgo ${ }^{3}$

Recebido em: 11 de junho de 2021. Aceito em: 21 de julho de 2021.

Publicado online em: 14 de setembro de 2021.

DOI: $10.37310 /$ ref.v90i2.2765

\section{Resumo}

Introdução: 0 Ministério da Defesa (MD) criou, em 2008, o Programa de Incorporação de Atletas de Alto Rendimento (PAAR), com o objetivo de fortalecer a equipe militar brasileira em eventos esportivos de alto nível e contribuir para o desenvolvimento do desporto nacional.

Objetivo: Avaliar o nível de satisfação dos atletas de alto rendimento das Forças Armadas com a qualidade da estrutura oferecida pelo PAAR, bem como conhecer se esses desportistas consideram o Programa importante para a melhoria do desempenho.

Métodos: Estudo de metodologia quantitativa e corte transversal, realizado no censo dos atletas integrantes do PAAR. Elaborou-se um questionário, autopreenchível, em plataforma on-line, composto por questões que visaram determinar o nível de satisfação dos atletas com a estrutura oferecida.

Resultados: Observou-se que 96,46\% dos participantes concordaram que o PAAR é ou foi importante para a melhoria de seu desempenho esportivo. Quanto às instalações desportivas, $63,3 \%$ classificaram os centros de treinamento como muito bons e $19,2 \%$ como bons, somando $82,2 \%$. Sobre o nível de satisfação dos atletas com o programa, cerca de $96,46 \%$ dos participantes da pesquisa estão satisfeitos ou

Pontos Chave

- Programa de Incorporação de Atletas de Alto Rendimento

(PAAR) tem por objetivo fortalecer o esporte militar e contribuir para o desenvolvimento do desporto nacional.

\section{- 96,46\% dos participantes} concordaram que o PAAR é ou foi importante para a melhoria de seu desempenho esportivo. - 84,4\% estavam muito satisfeitos e $12,0 \%$ estavam satisfeitos com o Programa, somando $96,4 \%$ dos atletas. muito satisfeitos com o programa. Oportunidades de melhoria foram apontados pelos participantes.

Conclusão: A satisfação dos atletas do PAAR com o Programa é muito alta. Considerando-se que proporciona grande suporte financeiro, instalações desportivas adequadas e apoio de saúde de qualidade. Os resultados apontaram que os seus integrantes consideram categoricamente o Programa como eficaz para possibilitar melhores condições de treinamento, o que reflete positivamente sobre o desempenho e, consequentemente, nos resultados esportivos.

Palavras-chave: esporte, atletas, alto rendimento, gestão, administração.

$\S$ Autor correspondente: Marcelo Lucena de Almeida - e-mail: lucenaalmeida@hotmail.com Afiliações: ${ }^{1}$ Comissão de Desportos do Exército (CDE), Rio de Janeiro, RJ, Brasil; ${ }^{2}$ Comissão Desportiva Militar do Brasil (CDMB), Brasília, DF, Brasil; ${ }^{3}$ Escola de Comando e Estado-Maior do Exército (ECEME), Rio de Janeiro, RJ, Brasil. 


\begin{abstract}
Introduction: In 2008, the Ministry of Defense (MD) created the Program for the Incorporation of HighPerformance Athletes (PIHPA), with the objective of strengthening the Brazilian military team in high-level sporting events and contributing to the development of national sport.

Objective: Assess the level of satisfaction of highperformance athletes in the Armed Forces with the quality of the structure offered by PIHPA, as well as knowing whether these athletes consider the Program important for improving performance.

Methods: Study of quantitative methodology and crosssectional, carried out in the census of athletes participating in the PIHPA. A self-administered questionnaire was developed on an online platform, consisting of questions that aimed to determine the level of satisfaction of athletes with the structure offered.
\end{abstract}

Results: It was observed that $96.46 \%$ of the participants agreed that the PIHPA is or was important for the improvement of their sports performance. As for sports facilities, $63.3 \%$ rated the training centers as very good and $19.2 \%$ as good, totaling $82.2 \%$. Regarding the level of satisfaction of athletes with the program, about $96.46 \%$ of survey participants are satisfied or very satisfied with the program. Opportunities for improvement were pointed out by the participants.

Conclusion: The satisfaction of PIHPA athletes with the Program is very high. Considering that it provides great financial support, adequate sports facilities, and quality health support. The results showed that its members categorically consider the Program as effective in enabling better training conditions, which reflects positively on performance and, consequently, on sports results.

\section{A importância do Programa de Atletas de Alto Rendimento (PAAR) das Forças Armadas para o desempenho de atletas brasileiros: um estudo transversal}

\section{Introdução}

As Forças Armadas do Brasil são um forte indutor e incentivador do Desporto Nacional nas manifestações esportivas previstas na Lei 9.615, de 24 de março de 1998, popularmente conhecida como a "Lei Pelé"(1). De acordo com Ribeiro(2) e Soeiro(3), a evolução da Educação Física no Brasil está fortemente ligada com as Forças Armadas e ao Treinamento Físico Militar. Isto porque a própria origem histórica do exercício físico remonta ao início das civilizações humanas, com os treinamentos para a guerra. Nesse contexto, é importante ressaltar que, historicamente, o ambiente militar é o principal difusor de influências relevantes para o desenvolvi- mento e metodização da Educação Física, sendo destacada a contribuição do Exército Brasileiro para o esporte no Brasil. De acordo com a literatura, os militares foram os pioneiros e tiveram grande influência na organização e no desenvolvimento inicial do conhecimento das práticas de exercício físico(3).

O Ministério da Defesa (MD), protagonista das estratégias do Desporto Militar Brasileiro, em 2008, criou o Programa de Incorporação de Atletas de Alto Rendimento (PAAR) que, inicialmente, visava fortalecer a equipe militar brasileira em eventos esportivos de alto nível. O Programa recebeu regulamentação específica em 2019, por 
meio da publicação da Portaria Normativa $\mathrm{N}^{\circ}$ 71/GM-MD, de 14 de agosto de 2019(4). No documento, foram definidos os objetivos para o PAAR. São eles:

"I - Representar o Ministério da Defesa e as Forças Armadas brasileiras em competições esportivas nacionais e internacionais;

II - Projetar positivamente a imagem das Forças Armadas brasileiras no País e no exterior;

III - Motivar a prática esportiva e a higidez física pelos militares e transferir conhecimento técnico científico da atividade desportiva para o militar; $e$

IV - Contribuir para o desenvolvimento do desporto nacional de rendimento, educacional, de participação e de formação."

O PAAR é composto por terceiros sargentos, cabos, soldados e marinheiros, convocados pelas Forças Armadas. Esses atletas possuem habilitações técnicas em atividade física e desporto de alto rendimento nas diversas modalidades. $\mathrm{Na}$ condição de militares temporários da ativa, têm direito a: soldo (salário), $13^{\circ}$ salário, férias, assistência médica e odontológica e instalações esportivas militares de alto padrão oferecidas pelas organizações militares: o Centro de Educação Física Almirante Adalberto Nunes, da Marinha do Brasil, o Centro de Capacitação Física do Exército e a Universidade da Força Aérea (UNIFA). Essa convocação é anual, renovável por até oito anos(4).

De acordo com os dados da Comissão Desportiva Militar do Brasil (CDMB) $(5,6)$, atualmente, 36 esportes participam do PAAR, sendo 28 (vinte e oito) modalidades olímpicas, 5 (cinco) modalidades não olím-

\footnotetext{
${ }^{1}$ Pentatlo Moderno: natação (200 m nado livre), esgrima, hipismo, tiro ao alvo e corrida através campo $(3.000 \mathrm{~m})$ (7).

${ }^{2}$ Triatlo: natação, ciclismo e corrida.

${ }^{3}$ Pentatlo Aeronáutico - O esporte foi idealizado no intuito de testar o condicionamento físico e a capacidade de um piloto abatido em solo inimigo sobreviver e regressar ao território amigo. Embora
}

picas e 3 (três) modalidades exclusivamente militares, reunindo um efetivo de aproximadamente 600 (seiscentos) atletas. Os esportes olímpicos atualmente apoiados pelo Programa são: atletismo, basquete, boxe, caratê, canoagem, ciclismo, escalada, esgrima, futebol, golfe, ginástica artística, hipismo, judô, levantamento de peso, lutas associadas, maratona aquática, nado sincronizado, natação, pentatlo moderno ${ }^{1}(7)$, remo, saltos ornamentais, taekwondo, tiro esportivo, tiro com arco, triatlo ${ }^{2}$, vela, voleibol e vôlei de praia. Os esportes não olímpicos são: futebol de praia (beach soccer), corrida rústica (cross-country), orientação, paraquedismo e salvamento aquático. Os esportes exclusivamente militares são: pentatlo aeronáutico ${ }^{3}(8,9)$, pentatlo militar(10,11) e pentatlo na$\operatorname{val}^{4}(11)$.

Desde a criação do PAAR, aproximadamente 1.000 atletas foram incorporados às Força Armadas e têm usufruído dos benefícios que o Programa fornece. Face à necessidade de se examinar os resultados do Programa, surgiu a questão: Será que os atletas estão satisfeitos com o Programa, levando em consideração as suas condições para o treinamento $\mathrm{e}$ performance / resultados alcançados?

Dessa forma, o objetivo deste estudo foi avaliar o nível de satisfação dos atletas de alto rendimento das Forças Armadas com a qualidade da estrutura oferecida pelo PAAR, bem como conhecer se esses desportistas consideram o Programa importante para a melhoria do seu desempenho.

$\S$ Autor correspondente: Marcelo Lucena de Almeida - e-mail: lucenaalmeida@hotmail.com Afiliações: ${ }^{1}$ Comissão de Desportos do Exército (CDE), Rio de Janeiro, RJ, Brasil; ${ }^{2}$ Comissão Desportiva Militar do Brasil (CDMB), Brasília, DF, Brasil; ${ }^{3}$ Escola de Comando e Estado-Maior do Exército (ECEME), Rio de Janeiro, RJ, Brasil. 


\section{Métodos}

\section{Desenho de estudo e amostra}

Estudo de caráter descritivo com metodologia quantitativa e corte transversal, realizado no censo dos atletas integrantes do PAAR. Foram convidados para participar do estudo 826 atletas de ambos os sexos, das Forças Armadas.

O critério de inclusão foi ser atualmente ou ter sido atleta integrante do PAAR. Os critérios de exclusão foram: não estar disponível para participar do estudo no período oferecido na plataforma on-line de participantes no estudo.

\section{Aspectos éticos}

Todos os princípios que regem a ética em pesquisa envolvendo seres humanos, constantes das Resoluções $n^{\circ} 466 / 2012$ e $n^{\circ}$ 510/2016 do Conselho Nacional de Saúde (CNS) do Ministério da Saúde (MS) foram observados e o Termo de Consentimento Livre e Esclarecido (TCLE) foi assinado.

\section{Instrumento}

O questionário é um método concreto para se obter informações, com custo razoável, garantindo o anonimato e que garante uniformidade e padronização dos dados(12). Dessa forma, para avaliar a percepção dos atletas quanto à qualidade do PAAR, foi elaborado um questionário autopreenchível, em plataforma on-line, composto por questões que visaram determinar o nível de satisfação dos atletas das Forças Armadas com o Programa (Anexo I).

O instrumento foi composto por 22 perguntas distribuídas em quatro partes. A $1^{\text {a }}$ parte destinou-se a identificar características do perfil do atleta. Foi composta por nove questões (Q1-Q9) que abordaram: a Força à qual o atleta pertencia; modalidade esportiva; idade; situação (integrante / ex-integrante); ano de ingresso / saída; e níveis de participações internacionais (Panamericanos / Mundiais / Jogos Olímpicos).

A $2^{\mathrm{a}}$ parte destinou-se a avaliar a percepção do atleta quanto ao nível de eficácia do PAAR. Foi composta por duas questões (Q10-Q11). A questão 10 era sobre a importância do PAAR em relação ao desempenho atlético, apresentando opções de resposta em escala de Likert: Discordo Totalmente / Discordo /Não Estou Decidido / Concordo / Concordo Totalmente). A questão 11 referiu-se ao grau de satisfação com o Programa, com respostas em escala de Likert: Nada Satisfeito / Pouco Satisfeito / Satisfeito / Muito Satisfeito / Totalmente Satisfeito.

A $3^{\text {a }}$ parte destinou-se aos atletas que participavam naquele momento do programa, portanto, não era para os exintegrantes. Composta por questões abertas, buscou-se dar oportunidade para que os atletas pudessem externar valores mais subjetivos em relação à percepção da qualidade a eles oferecida no PAAR. As perguntas (Q12-Q16) abordaram a participação do atleta no PAAR ou em outro programa / fonte de renda durante o período da pandemia de CoViD-19, para se avaliar a importância do Programa em relação aos recursos financeiros dos atletas durante o período da pandemia.

A $4^{\mathrm{a}}$ e última parte (Q17-Q22), levantou a utilização pelo atleta das instalações desportivas e do Serviço de Saúde, bem como avaliar sua percepção quanto à qualidade de ambos.

\section{Procedimento de coleta de dados}

A pesquisa teve caráter voluntário e sigiloso. Os participantes foram esclarecidos desses dois pontos no e-mail que encaminhou o link da pesquisa e reforçado no TCLE. Dessa forma, garantindo ao participante, mesmo antes do envio de suas respostas, que o sigilo das informações seria mantido. Ressalta-se que os participantes também estavam livres para escolher quais perguntas desejariam responder.

A pesquisa esteve disponível para resposta na plataforma durante 30 (trinta) dias, no período de $1^{\circ}$ a 30 de setembro de 2020. A pesquisa foi enviada para 826 atletas ou ex-atletas das Forças Armadas do PAAR, sendo que responderam a uma ou mais perguntas 424 indivíduos.

\section{Análise estatística}

Para análise dos dados foi verificada a frequência de respostas em cada questão e 
apresentada uma estatística descritiva dos achados.

\section{Resultados}

Dos 826 atletas das Forças Armadas convidados para participar do estudo, 424 $(51,33 \%)$ responderam o questionário. A média de idade foi de $28,78( \pm 5,73)$, sendo 367 atletas e 54 ex-atletas (03 não informaram sua situação no Programa). O questionário oferecia ao atleta a liberdade de responder ou não cada pergunta, assim, cada questão poderia apresentar um total de respostas recebidas distinto das demais.

\section{Situação profissional durante a pandemia}

Quanto à situação profissional dos atletas dentro e fora do PAAR (Q12-Q16), durante a pandemia de CoViD-19, destinou-se somente aos atletas atualmente vinculados ao Programa. Inicialmente, 375 atletas responderam sobre a importância do Programa na Pandemia de Covid-19 (Q12), sendo que 345 (92\%) consideraram o PAAR muito importante fazer parte do Programa, e 21 (5,6\%) importante. Nenhum militar assinalou a opção "não é importante".

$\mathrm{Na}$ Q13 foi perguntado se o atleta participava de outro programa de incentivo ao esporte, das 388 respostas, 251 (64,9\%) responderam que sim.

$\mathrm{Na}$ Q14 foi questionado se o atleta possuía outra fonte de renda além do proveniente das Forças Armadas, e das 383 respostas, $290(75,7 \%)$ responderam que sim e 93 informaram que mesmo antes da pandemia só tinham as Forças Armadas como fonte de renda.

$\mathrm{Na} \mathrm{Q} 15$, foi perguntado se o atleta perdeu algum patrocínio ou renda durante a Pandemia, sendo que das 378 respostas, 192 $(64,9 \%)$ responderam que sim.

$\mathrm{Na}$ Q16, das 243 respostas, $120(49,4 \%)$ responderam que o PAAR se tornou a única fonte de renda.

\section{Instalações esportivas}

Quanto à qualidade das instalações desportivas oferecidas nos Centros de Treinamento (Q17), dos 422 que respon- deram ao questionamento, cerca de 267 $(63,3 \%)$ classificaram-nos como muito bons e $81(19,2 \%)$ como bons, que somados representaram $82,2 \%$ dos atletas respondentes.

Em relação a considerar que esses Centros de Treinamento auxiliaram na melhora de seu desempenho esportivo (performance) (Q18), 412 responderam, sendo que $86 \%$ consideraram que sim, conforme exibe-se no Gráfico 1.

\section{Serviço de Saúde}

Quanto ao atendimento no Serviço de Saúde (médicos, fisioterapeutas, massoterapeutas, nutricionistas, dentistas e psicólogos) (Q19), dos 418 que responderam, $19,6 \%$ informaram que nunca utilizaram o serviço de saúde.

Dos 409 atletas que responderam à Q20, a maioria dos atletas $(82,4 \%)$ informou que as instalações de saúde dos Centros de Treinamento Militar (CTM) auxiliam na melhoria da performance desportiva (Gráfico 2).

\section{Programa de Atletas de Alto Rendimento (PAAR): desempenho esportivo e nível de satisfação}

Em relação à importância do PAAR para a melhoria do desempenho esportivo (performance) (Q10), dos 421 atletas que responderam, $83,6 \% \quad$ concordaram totalmente e $13,3 \%$ concordaram, que juntos somaram $96,46 \%$ e afirmaram que o Programa é/foi muito importante para a melhoria de seu desempenho (performance) em competições, e $0,4 \%$ discordaram da afirmativa (Gráfico 3).

Quanto ao nível de satisfação dos atletas com o Programa (Q11), dos 424 respondentes, $409 \quad(96,4 \%)$ informaram estar totalmente satisfeitos $(84,4 \%)$ e muito satisfeitos $(12 \%)$. Os que informaram estar nada ou pouco satisfeitos somaram 2,6\%, conforme se observa no Gráfico 4 .

$\mathrm{Na} 3^{\mathrm{a}}$. parte do questionário, foi possível observar que satisfação dos atletas foi evidenciada. As questões ofereciam resposta do tipo aberta configurando-se em campo de opinião livre dos entrevistados,

$\S$ Autor correspondente: Marcelo Lucena de Almeida - e-mail: lucenaalmeida@hotmail.com Afiliações: ${ }^{1}$ Comissão de Desportos do Exército (CDE), Rio de Janeiro, RJ, Brasil; ${ }^{2}$ Comissão Desportiva Militar do Brasil (CDMB), Brasília, DF, Brasil; ${ }^{3}$ Escola de Comando e Estado-Maior do Exército (ECEME), Rio de Janeiro, RJ, Brasil. 
no qual foram deixados diversos relatos que transcrevem a emoção e a gratidão de vários atletas. Dos 262 comentários deixados, $98,5 \%$ exibiram conteúdos positivos. A seguir, destacam-se alguns dos comentários:

As instalações desportivas dos Centros de Treinamento Militares auxiliaram na melhora da sua performance esportiva?

412 respostas

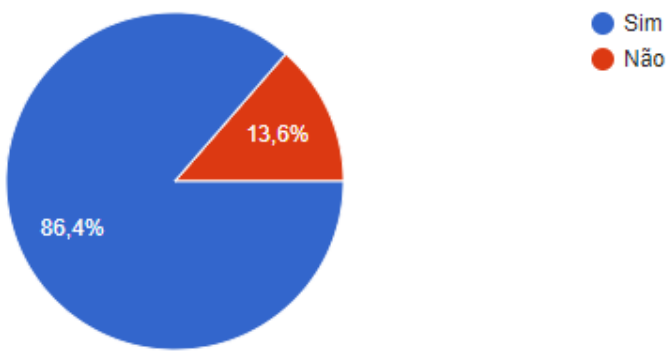

Gráfico 1 - Importância das instalações desportivas militares para o desempenho esportivo.

As instalações de saúde dos Centros de Treinamento Militares auxiliaram na melhora da sua performance esportiva?

409 respostas

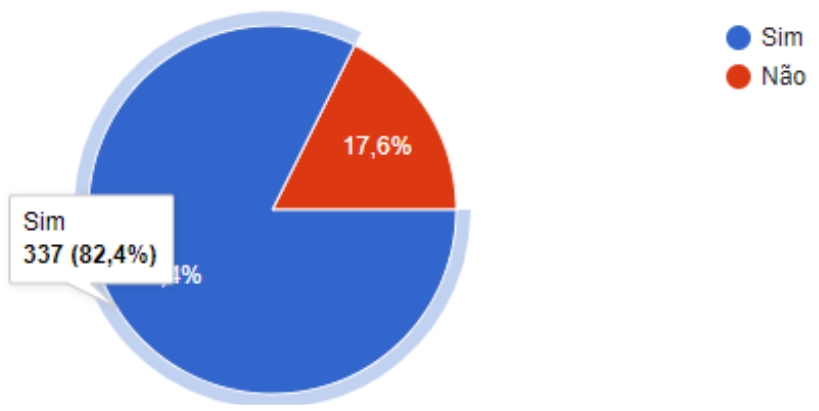

Gráfico 1 - Importância das instalações de saúde para o desempenho esportivo.

Em uma escala onde: 1 - discordo totalmente; 2 - discordo; 3 - não estou decidido; 4 concordo; e 5 - concordo totalmente, podemos afirmar que o PAAR é/foi muito importante para a melhoria da sua performance em competições.

421 respostas

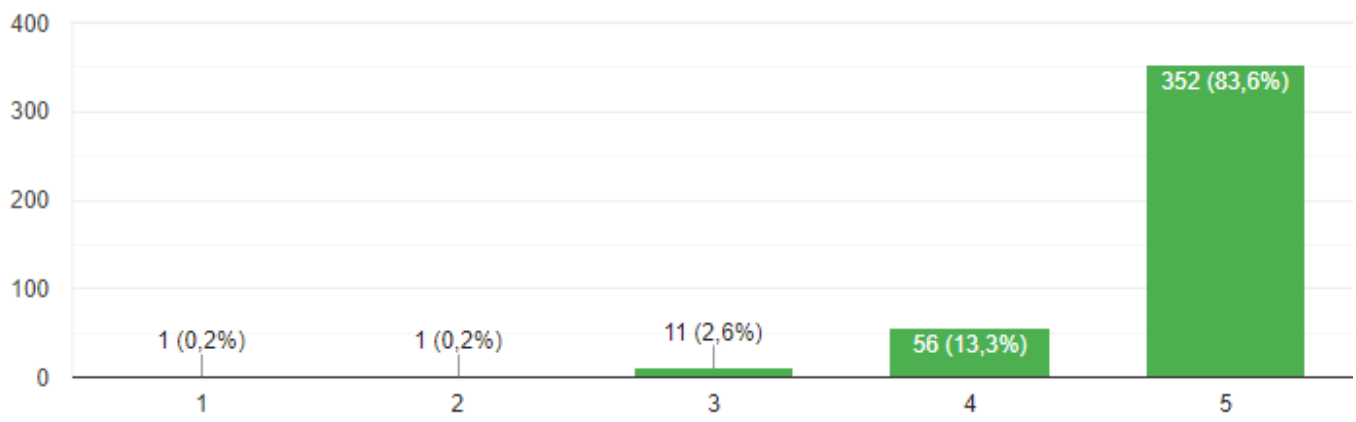


Gráfico 3 - Importância do PAAR na melhoria do desempenho esportivo.

Em uma escala onde: 1 - nada satisfeito, 2 - pouco satisfeito, 3 - satisfeito, 4 - muito satisfeito e 5 - totalmente satisfeito, qual o seu indice de satisfação com o referido Programa?

424 respostas

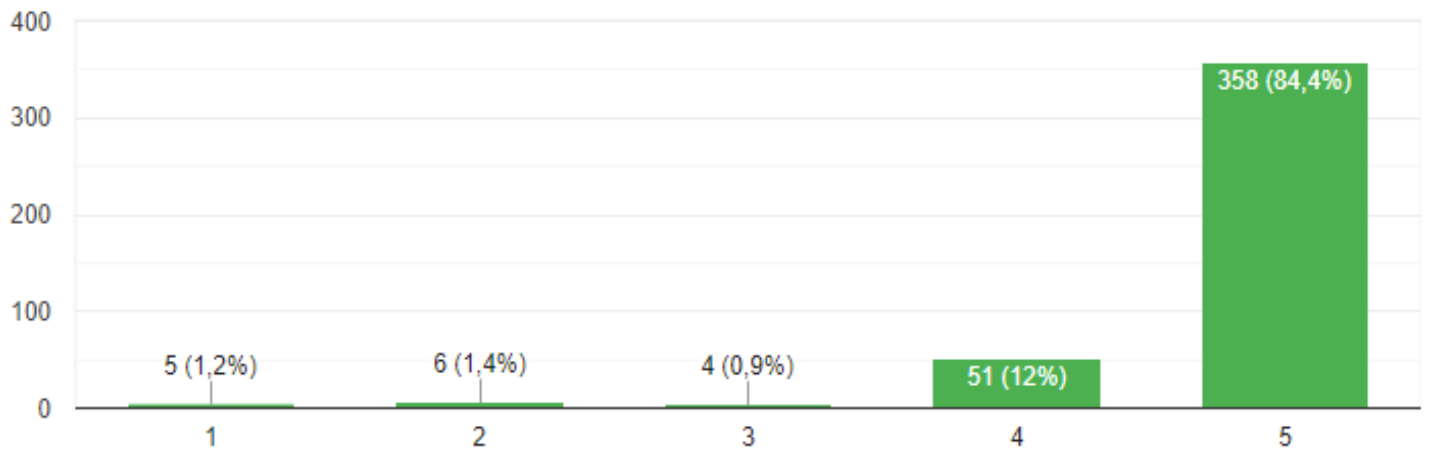

Gráfico 4 - Nível de satisfação dos Atletas Militares com o PAAR.

As questões Q21 e Q22 ofereciam resposta do tipo aberta configurando-se em campos de opinião livre dos entrevistados. $\mathrm{Na}$ Q21, foram deixados diversos relatos que transcreveram a emoção e a gratidão de vários atletas ao Programa. Dos 262 comentários deixados, 98,5\% exibiram conteúdos positivos. A seguir, destacam-se alguns dos comentários:

"O programa foi de grande valia na minha vida, pois além de ser fundamental para minha evolução como atleta, me fez um ser humano melhor e um homem mais responsável."

"Dando suporte de treinamento, saúde e financeiro de qualidade, o PAAR é um programa de excelência que serve de modelo nacional para diversas entidades. Ele oferece o que é de essencial para que os atletas rendam mais, podendo se dedicar somente ao esporte e, naturalmente, aumentar sua performance.'

"O PAAR tem uma importância enorme no crescimento dos atletas, tanto na parte financeira quanto na estrutura que nos oferece."

"Foi pra mim uma excelente experiência, guardo com muito carinho e orgulho ter feito parte do programa e quem sabe um dia ter condições de fazer parte de alguma outra forma!"

Em relação às oportunidades de melhorias no PAAR na percepção dos atletas (Q22), os resultados indicaram os seguintes aspectos:

Possibilidade de proporcionar uma dieta diferenciada para os atletas, que normalmente recebem alimentação similar aos demais militares;

Maior participação em treinamentos e competições no exterior, uma vez que alguns atletas consideram insuficiente a quantidade atual; e

$>$ A criação de um plano póscarreira para os atletas.

\section{Discussão}

Até o presente momento, analisando a literatura, identificou-se que este foi $o$ primeiro estudo a examinar a qualidade de centros de excelência de treinamento esportivo, dentre diversos programas existentes no país. Os principais resultados

$\S$ Autor correspondente: Marcelo Lucena de Almeida - e-mail: lucenaalmeida@hotmail.com Afiliações: ${ }^{1}$ Comissão de Desportos do Exército (CDE), Rio de Janeiro, RJ, Brasil; ${ }^{2}$ Comissão Desportiva Militar do Brasil (CDMB), Brasília, DF, Brasil; ${ }^{3}$ Escola de Comando e Estado-Maior do Exército (ECEME), Rio de Janeiro, RJ, Brasil. 
foram que, para maioria dos atletas participantes do PAAR das Forças Armadas, o nível de satisfação com o Programa foi indicado como totalmente satisfeito $(84,4 \%)$ ou muito satisfeito $(12 \%)$, sendo que apenas $2,6 \%$ consideraram-se pouco ou nada satisfeitos. Além disso, 96,46\% consideraram que o programa é/foi muito importante para a melhoria de seu desempenho esportivo, sendo que apenas $0,4 \%$ consideram o Programa pouco ou nada importante.

No esporte de alto rendimento, a melhoria do desempenho em competições é o grande objetivo dos atletas. Na busca por melhores resultados, atletas e treinadores têm procurado ir além da preparação física, técnica e tática, investindo em outras áreas associadas ao treinamento esportivo, demonstrando uma tendência à interdisciplinaridade(13). Da mesma forma, a busca constante pelo aumento no desempenho é o grande objetivo dos atletas de alto rendimento do PAAR. Nesse contexto, é de grande relevância mensurar os benefícios promovidos pelo PAAR sob a perspectiva dos atletas, o que pode contribuir para o aprimoramento das estruturas e serviços oferecidos pelo Programa. Assim, os resultados do presente estudo, quanto à contribuição do PAAR para a melhoria do desempenho esportivo indicada pela maioria dos atletas - cerca de 97\% (Gráfico 3), apontam para o sucesso do Programa.

O desenvolvimento de políticas esportivas para instalações esportivas é um dos fatores que contribuem para o sucesso esportivo (14). Nessa perspectiva, os três CTM de referência nacional (CEFAN, CCFEx e UNIFA) possuem instalações desportivas multidisciplinares de excelência, alojamentos próximos às arenas, laboratórios de pesquisa, equipes médicas disponíveis, locais para refeições, entre outras facilidades.

O esporte profissional é caracterizado pela busca pelo melhor rendimento possível do atleta, envolvendo uma equipe multidisciplinar realizando um trabalho interdisciplinar, com o objetivo de elevar a capacidade do esportista(15). Um dos fatores desfavoráveis ao desempenho que, muitas vezes, o atleta de alto rendimento precisa enfrentar é a ocorrência de dor. A dor é considerada um dos eventos que mais limita o desempenho de um atleta de alto rendimento, além de ser presença frequente na vida desses desportistas(16,17). Nesse contexto, os centros de treinamento das Forças Armadas possuem equipes de saúde compostas por médicos, enfermeiros, fisioterapeutas e massoterapeutas que podem contribuir para diminuir e prevenir os efeitos das lesões nos atletas do PAAR. Poucos atletas utilizaram os serviços dos profissionais de saúde oferecidos no Programa, o que é esperado para uma população aparentemente saudável.

A maioria dos atletas $(86,4 \%)$ considerou que as instalações do serviço de saúde contribuíram positivamente para seu desempenho, sendo que apenas 19,2\% afirmaram ter precisado de atendimento médico. Esses resultados indicam que as estruturas do serviço de saúde nos CTM tanto em termos de instalações, quanto de pessoal apresentam-se em condições bastante satisfatórias.

As questões abertas com respostas de opinião livre realizada no presente estudo mostraram que a satisfação dos atletas ficou ainda mais evidente no campo de opinião livre dos entrevistados. Foram observados diversos relatos que transcreveram a emoção e gratidão de vários atletas.

A taxa de resposta dos atletas ao questionário de $51,33 \%$, poderia ser baixa quando se pretendia avaliar o censo dos atletas participantes do programa. Entretanto, observa-se que estudos de pesquisa em administração, publicados em jornais altamente qualificados, frequentemente, reportaram taxas de resposta entre $40 \%$ e $60 \%(18-22)$. Mellahi e Harris(21) em estudo sobre métodos de pesquisa científica na área da gestão, discutiram que, para área da gestão em recursos humanos, uma taxa $50 \%$ deve ser considerada boa, como foi o caso da taxa de resposta obtida no presente estudo.

O PAAR proporciona aos atletas integrantes do Programa um grande suporte financeiro, instalações desportivas adequadas e apoio de saúde de qualidade. Isso 
reverbera para que os seus integrantes considerem categoricamente o programa como eficaz para possibilitar melhores condições de treinamento, o que, segundo os achados do presente estudo, se reflete no desempenho em resultados esportivos.

Os achados do presente estudo vão ao encontro do estudo de Costa(23), que concluiu que o PAAR possui papel importante dentro da política esportiva brasileira e apresenta-se como uma importante estratégia para o desenvolvimento do desporto de alto rendimento no Brasil, podendo servir de base para futuras políticas públicas. A constatação de o Programa contribui para melhorar o desempenho dos atletas de alto rendimento do Brasil, considera-se que o PAAR cumpre um de seus principais objetivos, o de contribuir para o desenvolvimento do desporto nacional. Com isso, estimula-se a manutenção do Programa e o incremento de sua divulgação, visando estimular a participação de jovens com pendor esportivo para bem representar as Forças Armadas e o Brasil em eventos nacionais e internacionais, contribuindo para a promoção do sentimento de pertencimento e fomento ao desporto nacional.

\section{Pontos fortes e limitações do estudo}

Um ponto forte do estudo reside no fato de ter sido o primeiro que buscou quantificar o nível de satisfação dos atletas militares participantes de programa de incentivo ao esporte nacional. Atualmente, além do PAAR, no Brasil, há outros programas de governo de incentivo ao desporto de alto rendimento, como o Bolsa Atleta e o Bolsa Pódio e não foram encontrados estudos similares que tivessem buscado quantificar a satisfação dos atletas com esses programas.

Uma limitação do estudo foi que as análises não foram feitas segundo sexo, o que poderia esclarecer se haveria diferenças em percepção entre os atletas dos times masculino e feminino, o que se recomenda que seja realizado em estudos futuros.

\section{Conclusão}

O objetivo do presente estudo foi apresentar à comunidade científica o PAAR e avaliar o nível de satisfação dos atletas das Forças Armadas com a qualidade dos serviços e instalações oferecidos no Programa, bem como conhecer se esses desportistas o consideraram importante para a melhoria do seu rendimento.

Os resultados encontrados mostraram que o nível de satisfação dos atletas com o PAAR é excelente, bem como é elevado o nível de satisfação com os centros de treinamento das Forças Armadas, em termos de estruturas físicas (esportivas, alojamentos e de serviço de saúde) e foram considerados pelos atletas como fatores importantes para o aumento do desempenho desportivo.

\section{Declaração de conflito de interesses}

Não há nenhum conflito de interesses em relação ao presente estudo.

\section{Declaração de financiamento}

Não houve financiamento nesse estudo.

\section{Referências}

1. Brasil. Lei no. 9.615 de 24 de março de 2019. [Online] 9.615 Mar 24, 2019. Available from: http://www.planalto.gov.br/ccivil_03/leis/ 19615consol.htm [Accessed: 7th July 2021]

2. Ribeiro AM. Contribuições da Missão Militar Francesa para o desenvolvimento do desporto no Exército Brasileiro (Parte I). Revista de Educação Física / Journal of Physical Education. [Online] 2009;78(146). Available from: doi:10.37310/ref.v78i146.316 [Accessed: 7th July 2021]

3. Soeiro R. Escola de Educação Física do Exército - Legado ao esporte nacional 1933-2018. Rio de Janeiro: Biblioteca do Exército; 2019. 232 p.

4. Ministério da Defesa. Portaria Normativa no. $071 \mathrm{GM} / \mathrm{MD}$ de 14 de agosto de 2019. [Online] 71 Aug 14, 2019. Available from: https://www.in.gov.br/en/web/dou//portaria-normativa-n-71/gm-md-de-14-

\footnotetext{
$\S$ Autor correspondente: Marcelo Lucena de Almeida - e-mail: lucenaalmeida@hotmail.com Afiliações: ${ }^{1}$ Comissão de Desportos do Exército (CDE), Rio de Janeiro, RJ, Brasil; ${ }^{2}$ Comissão Desportiva Militar do Brasil (CDMB), Brasília, DF, Brasil; ${ }^{3}$ Escola de Comando e Estado-Maior do Exército (ECEME), Rio de Janeiro, RJ, Brasil.
} 
de-agosto-de-2019-212908744 [Accessed: 22nd July 2021]

5. Ministério da Defesa. Comissão Desportiva Militar completa 65 anos e contribui com fortalecimento do esporte nacional. [Online] Notícias - Comissão Desportiva Militar completas 65 anos. Available from: https://www.gov.br/defesa/pt-br/centraisde-conteudo/noticias/comissaodesportiva-militar-completa-65-anos-econtribui-com-fortalecimento-do-esportenacional-1 [Accessed: 7th July 2021]

6. Ministério da Defesa. Departamento de Desporto Militar. [Online] Available from: https://www.gov.br/defesa/ptbr/assuntos/desportomilitar/departamento-de-desporto-militar [Accessed: 7th July 2021]

7. Física R de E. Pentatlo Moderno. Revista de Educação Física / Journal of Physical Education. 1948;17(4): 4-4.

8. Ministério da Defesa. Conheça os militares brasileiros que se destacaram em Campeonato Mundial de Pentatlo Aeronáutico. [Online] Notícias - Conheça os militares brasileiros que se destacaram em Campeonato Mundial de Pentatlo Aeronáutico. Available from: https://www.gov.br/defesa/pt-br/centraisde-conteudo/noticias/ultimasnoticias/conheca-os-militares-brasileirosse-destacaram-em-campeonato-mundialde-pentatlo-aeronautico [Accessed: 7th July 2021]

9. Força Aérea Brasileira. Equipe Brasileira de Pentatlo Aeronáutico vence competição internacional - Força Aérea Brasileira. [Online] Esporte: Equipe brasileira de Pentatlo Aeronáutico vence competição internacional. Available from: https://www.fab.mil.br/noticias/mostra/34 202/ESPORTE\%20-

$\%$ 20Equipe $\% 20$ Brasileira $\% 20 \mathrm{de} \% 20$ Pent atlo $\% 20$ Aeron $\% \mathrm{C} 3 \%$ A 1 utico $\% 20$ vence $\%$ 20competi\%C3\%A7\%C3\%A3o\%20intern acional [Accessed: 7th July 2021]

10. Carvalho A de. Pentatlo Militar. Revista de Educação Física / Journal of Physical Education. 1958;27(1_2): 2-2.

11. Brasil EB de C. Conheça os esportes dos Jogos Mundiais Militares. [Online] Agência Brasil. Available from: https://agenciabrasil.ebc.com.br/esportes/ noticia/2019-10/conheca-os-esportes-dosjogos-mundiais-militares [Accessed: 7th July 2021]

12. Chaer G, Diniz RRP. A técnica do questionário na pesquisa educacional. 2011;7(7): 16.

13. Brandt R, Viana M da S, Segato L, Kretzer FL, Carvalho T de, Andrade A. Relações entre os estados de humor e o desempenho esportivo de velejadores de alto nível. Psicologia: teoria e prática. 2011;13(1): 117-130.

14. Bastos F, Mazzei L, Bohme M. Centros de Treinamento Esportivo Para o Esporte de Alto Rendimento no Brasil: Um Estudo Preliminar. Centro Esportivo Virtual. 2012;20(1): 1575-1584.

15. Galatti LR, Paes RR, Darido SC. Pedagogia do Esporte: livro didático aplicado aos Jogos Esportivos Coletivos. Motriz: Revista de Educação Física. [Online] Universidade Estadual Paulista; 2010;16: 751-761. Available from: doi:10.5016/1980-6574.2010v16n3p751

16. Silva EM da, Rabelo I, Rubio K. A dor entre atletas de alto rendimento. Revista Brasileira de Psicologia do Esporte. Associação Brasileira de Psicologia do Esporte; 2010;3(1): 79-97.

17. Hainline B, Turner JA, Caneiro JP, Stewart M, Moseley GL. Pain in elite athletesneurophysiological, biomechanical, and psychosocial considerations: a narrative review. British Journal of Sports Medicine. [Online] BMJ Publishing Group Ltd and British Association of Sport and Exercise Medicine; 2017;51(17): 1259-1264. Available from: doi:10.1136/bjsports2017-097890

18. Baruch Y. Response Rate in Academic Studies - A Comparative Analysis. Human Relations. [Online] 1999;52(4): 421-438. Available from: doi:10.1023/A:1016905407491

19. Baruch Y, Holtom BC. Survey response rate levels and trends in organizational research. Human Relations. [Online] SAGE Publications Ltd; 2008;61(8): 1139-1160. Available from: doi:10.1177/0018726708094863

20. Anseel F, Lievens F, Schollaert E, Choragwicka B. Response Rates in Organizational Science, 1995-2008: A 
Meta-analytic Review and Guidelines for Survey Researchers. Journal of Business and Psychology. [Online] 2010;25(3): 335-349. Available from: doi:10.1007/s10869-010-9157-6

21. Mellahi K, Harris LC. Response Rates in Business and Management Research: An Overview of Current Practice and Suggestions for Future Direction. British Journal of Management. [Online] 2016;27(2): 426-437. Available from: doi:10.1111/1467-8551.12154

22. Pielsticker DI, Hiebl MRW. Survey Response Rates in Family Business Research. European Management Review. [Online] 2020;17(1): 327-346. Available from: doi:10.1111/emre.12375

23. Costa C. Exército e o PAAR : processos de militarização no esporte nacional. [Online] [Dissertação de Mestrado] [Curitiba, PR]: Universidade Federal do Paraná; 2020. Available from: https://acervodigital.ufpr.br/handle/1884/6 7327 [Accessed: 8th July 2021] 


\section{Anexo I}

\section{QUESTIONÁRIO}

O Questionário a seguir tem o objetivo de levantar o nível de eficácia do PAAR para a melhoria da performance dos atletas de alto rendimento do Brasil e a contribuição desse projeto para o Desporto Nacional. Ele está dividido em 04 partes.

\section{$1^{a}$ parte}

Esta parte é destinada ao levantamento do perfil do atleta.

1) Força: $\square$ Marinha $\square$ Exército $\square$ Força Aérea

2) Modalidade:

3) Idade:

4) Situação:

5) Ano de ingresso no PAAR?

6) Ano de saída do PAAR, se for o caso?

7) O Sr./Sra. participou de Jogos Pan-americanos após o ingresso no PAAR?

Sim

Não

8) O Sr./Sra. participou de Campeonatos Mundiais (civis) da sua modalidade após o ingresso no PAAR?

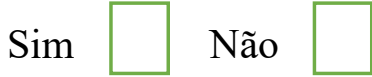

9) O Sr./Sra. participou de Jogos Olímpicos após o ingresso no PAAR?

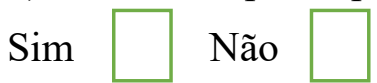

\section{$2^{\text {a }}$ Parte}

Esta parte tem a finalidade de identificar o nível de eficácia do PAAR.

10) Em uma escala onde: 1 - discordo totalmente; 2 - discordo; 3 - não estou decidido; 4 concordo; e 5 - concordo totalmente, podemos afirmar que o PAAR é/foi muito importante para a melhoria da sua performance em competições.

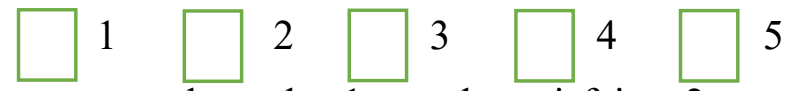

11) Em uma escala onde: 1 - nada satisfeito, 2 - pouco satisfeito, 3 - satisfeito, 4 - muito satisfeito e 5 - totalmente satisfeito, qual o seu índice de satisfação com o referido Programa?

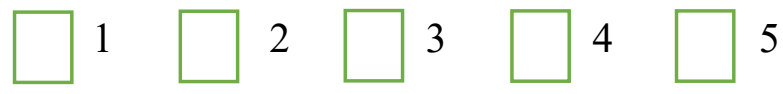

\section{$3^{\text {a }}$ Parte}

Esta parte é destinada somente para quem integra o PAAR atualmente. 
12) Em uma escala onde: 1 - não é importante; 2 - às vezes é importante; 3 - moderado; 4 importante; 5 - muito importante, como você classifica a importância de fazer parte do PAAR, durante o período de pandemia provocado pela Covid-19?
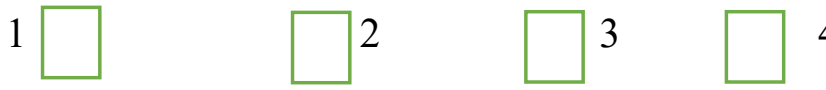

4

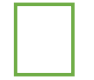

13) Você faz parte de algum outro programa governamental de incentivo ao esporte de rendimento?

Sim. Qual(is)

Bolsa Atleta

Bolsa Pódio

Outro

Não

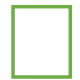

14) Antes do período de pandemia provocado pela COVID-19 o senhor (a) possuía outra fonte de renda além do proveniente das Forças Armadas?

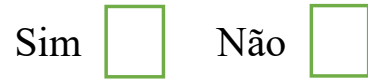

15) Caso tenha respondido SIM na pergunta anterior, durante o período de pandemia provocado pela COVID-19 o senhor(a) perdeu ou teve reduzida alguma fonte de renda proveniente de patrocínios de outras empresas ou instituições?

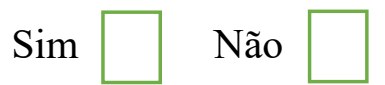

16) Caso tenha respondido SIM na pergunta anterior, a remuneração proveniente das Forças Armadas se tornou sua única fonte de renda?

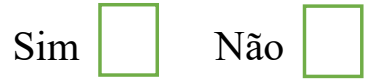

\section{$4^{\mathrm{a}}$ Parte}

Essa parte é destinada ao atleta postar sua opinião ou sugestões para o PAAR.

17) Como o senhor (a) classifica as instalações desportivas (quadras, piscinas, pistas, campos, ginásios, academias etc.) utilizados por sua modalidade nos Centros de Treinamento Militares?

Nunca utilizei $\square$ Ruins $\square$ Adequadas $\square$ Boas $\square$ Muito boas $\square$

18) As instalações desportivas dos Centros de Treinamento Militares auxiliaram na melhora da sua performance esportiva?

$\operatorname{Sim}$

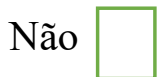

19) Como o senhor (a) classifica o serviço de saúde (médicos, fisioterapeutas, massoterapeutas, nutricionistas etc.) utilizados por sua modalidade nos Centros de Treinamento Militares?

Nunca utilizei.

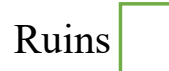

Adequadas

Boas

Muito boas

20) As instalações de saúde dos Centros de Treinamento Militares auxiliaram na melhora da sua performance esportiva?

Sim

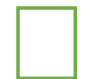

Não

$\S$ Autor correspondente: Marcelo Lucena de Almeida - e-mail: lucenaalmeida@hotmail.com Afiliações: ${ }^{1}$ Comissão de Desportos do Exército (CDE), Rio de Janeiro, RJ, Brasil; ${ }^{2}$ Comissão Desportiva Militar do Brasil (CDMB), Brasília, DF, Brasil; ${ }^{3}$ Escola de Comando e Estado-Maior do Exército (ECEME), Rio de Janeiro, RJ, Brasil. 
21) O senhor (a) teria sugestões para a melhorar o Programa?

22) Deixe aqui sua opinião sobre o Programa de Incorporação de Atletas de Alto Rendimento.

23) Declaro estar esclarecido dos seguintes pontos (TERMO DE CONSENTIMENTO LIVRE E ESCLARECIDO):

a) O Trabalho tem por finalidade quantificar e qualificar o nível de eficácia do PAAR para a melhora da performance dos atletas de alto rendimento do Brasil e a contribuição desse Programa para o Desporto Nacional;

b) A minha participação na pesquisa é fundamental para que os objetivos do trabalho sejam alcançados, sendo responsável por responder o questionário eletrônico com informações sobre o PAAR;

c) A pesquisa será realizada de 01 a 30 de setembro de maneira eletrônica, quando poderei responder aos questionamentos sobre o Programa.

d) Ao participar desse trabalho estarei contribuindo para a eficácia do PAAR e para a melhoria do PAAR;

e) Não terei nenhuma despesa no trabalho, bem como não receberei nenhum valor econômico a receber;

f) Minha participação será totalmente sigilosa, assegurando assim minha privacidade.

g) Qualquer dúvida poderei entrar em contato com os pesquisadores.

h) Ao enviar as respostas, estarei autorizando a utilização desses dados para trabalhos científicos.

\section{Enviar}

